\title{
Coexistent systemic amyloidosis, multiple myeloma, and refractory anemia with ringed sideroblasts in a previously untreated patient
}

\author{
Sangeeta Mehendale, Pritesh Patel, Valerie Lindgren \\ Damiano Rondelli. Suiata Gaitonde
}

\begin{abstract}
Introduction: We report the first case of coexistent systemic amyloidosis, plasma cell myeloma (MM), and myelodysplastic syndrome (refractory anemia with ringed sideroblasts:MDS-RARS). Case Report: Our patient was diagnosed with amyloidosis of the liver, stomach and tongue following which a bone marrow biopsy was performed. Review of the bone marrow biopsy revealed the presence of two distinct entities that have rarely been detected together; MM and MDS-RARS. There was no history of prior radiation or chemotherapy. Conclusion: Concurrent de novo disease processes involving both myeloid and lymphoid cell lines may not be as rare as they seem. Cytopenias in patients with MM must trigger a thorough investigation for an associated concurrent myeloid disorder. Presence of concurrent MDS would influence the choice of therapy and these patients need to be watched closely for progression of the myeloid disorder.
\end{abstract}

${ }^{1}$ Sangeeta Mehendale, ${ }^{2}$ Pritesh Patel, ${ }^{1}$ Valerie Lindgren 2Damiano Rondelli, 'Sujata Gaitonde

Affiliations: ${ }^{1}$ Department of Pathology, University of Illinois, Chicago, USA; ${ }^{2}$ Department of Medicine, University of Illinois, Chicago, USA.

Corresponding Author: Sujata Gaitonde, University of Illinois at Chicago, College of Medicine, Department of Pathology (MC 847), 840 South Wood Street; Room 110 CSN, Chicago, IL 60612; USA; Ph: 312996 4206,

Fax: 312413 0156; E-mail: sgaitond@uic.edu

Received: 25 August 2010

Accepted: 08 October 2010

Published: 28 February 2011
Keywords: Myeloma, Myelodysplastic syndrome, De novo

$* * * * * * * * *$

Mehendale S, Patel P, Lindgren V, Rondelli D, Gaitonde S. Coexistent systemic amyloidosis, multiple myeloma, and refractory anemia with ringed sideroblasts in a previously untreated patient. International Journal of Case Reports and Images 2011;2(2):1-4.

$$
* * * * * * * * * \text {. }
$$

doi:10.5348/ijcri-2011-02-17-CR-1

\section{INTRODUCTION}

Myelodysplastic syndrome (MDS) occurring during the course of MM generally results from administration of alkylating agents and is usually clonally unrelated to the preexisting multiple myeloma (MM). Therapy related MDS carries a poor prognosis due to a progressive course and resistance to conventional treatment. Four to ten percent of patients with MM develop MDS as a complication of therapy [1]. Here we report a case of coexistent systemic amyloidosis, MM and de-novo MDS which were present at the time of diagnosis in a patient with no history of chemotherapy or radiation therapy. MM has been reported in association with chronic lymphocytic leukemia, myeloproliferative neoplasms and acute leukemia [1]. Association of MM with de novo MDS is rare. They should be treated like any other multiple myeloma case.

\section{CASE REPORT}

A 74-year-old African-American man was admitted 
to our institution with a three month history of progressive generalized weakness accompanied by a 2olb weight loss and progressive exertional dyspnea. During this time the patient had been treated for symptomatic anemia with red cell transfusion on one occasion. He had also noted intermittent bleeding from his mouth. One week prior to presentation he developed jaundice. Physical examination revealed marked icterus. The oral cavity contained bilateral buccal mucosal lesions. The left buccal mucosa contained a superficial ulcerated lesion with irregular borders. The right buccal surface had an irregular ashen gray elevated lesion, $2 \mathrm{~cm} \mathrm{x} 1 \mathrm{~cm}$ in size. Abdominal examination showed non-pulsatile, nontender hepatomegaly.

Initial investigations revealed a mild microcytic, hypochromic anemia. The peripheral blood smear showed a mixed population of hypochromic and normochromic cells, with marked anisopoikilocytosis consisting of spherocytes, macrocytes, ovalocytes, teardrops and target cells, with few to moderate polychromatic forms and basophilic stippling. Serum iron levels were normal and serum ferritin was elevated at $410 \mathrm{ng} / \mathrm{ml}$. Liver function tests were remarkable for total bilirubin $14.9 \mathrm{mg} / \mathrm{dl}$, alkaline phosphatase $581 \mathrm{u} / \mathrm{l}$, AST $233 \mathrm{u} / \mathrm{l}$, ALT $241 \mathrm{u} / \mathrm{l}$, total protein $7.1 \mathrm{~g} / \mathrm{dl}$ and albumin $2.3 \mathrm{~g} / \mathrm{dl}$. An esophagealgastro-duodenal endoscopy showed gastric and duodenal ulcers and marked gastric wall thickening concerning for an infiltrative process such as linitis plastica. Abdominal imaging showed no evidence of biliary obstruction.

The patient underwent biopsies of the liver as well as of the oral and gastric lesions. Liver biopsy showed a panlobular perisinusoidal deposition of amyloid. (Figure 1) The gastric biopsy showed an expansion of lamina propria with an acellular, amorphous eosinophilic material suggestive of amyloid. The biopsy of the tongue showed similar morphology with infiltrative amorphous material, suggestive of amyloidosis. Congo red stain was positive in the gastric biopsy.

Bone marrow biopsy showed a hypercellular marrow for age $(70 \%)$ with trilineage hematopoiesis and erythroid hyperplasia (M: E ratio 0.9:1). (Figure 2) Salient findings included $31 \%$ ringed sideroblasts (Figure 3), slightly increased marrow iron stores, dyserythropoiesis and 33\% atypical marrow plasmacytosis that was confirmed by $\mathrm{CD}_{13} 8$ immunostain on the core biopsy. (Figure 4) Marrow blast count was not increased (3\%). Bone marrow core biopsy demonstrated a thickened vessel wall with focal area of amorphous eosinophilic material suggestive of amyloid deposition. Final bone marrow diagnosis was concomitant MM and low grade MDS (RARS) with systemic amyloidosis. Standard cytogenetic studies showed a normal male karyotype and FISH analysis for $T P_{53}$ deletion was negative.

Urine protein electrophoresis showed a monoclonal kappa light chain with $3.7 \mathrm{~g} /$ day of kappa light chains in the urine. Although serum immunoglobulins

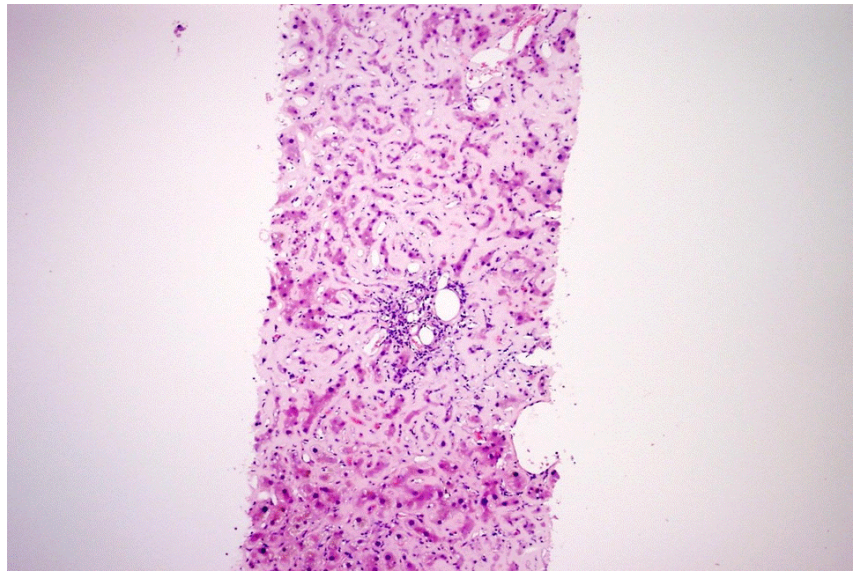

Figure 1: Core liver biopsy demonstrating homogenous pink amyloid deposition in the peri-sinusoidal / space of Disse. (H\&E, x40)

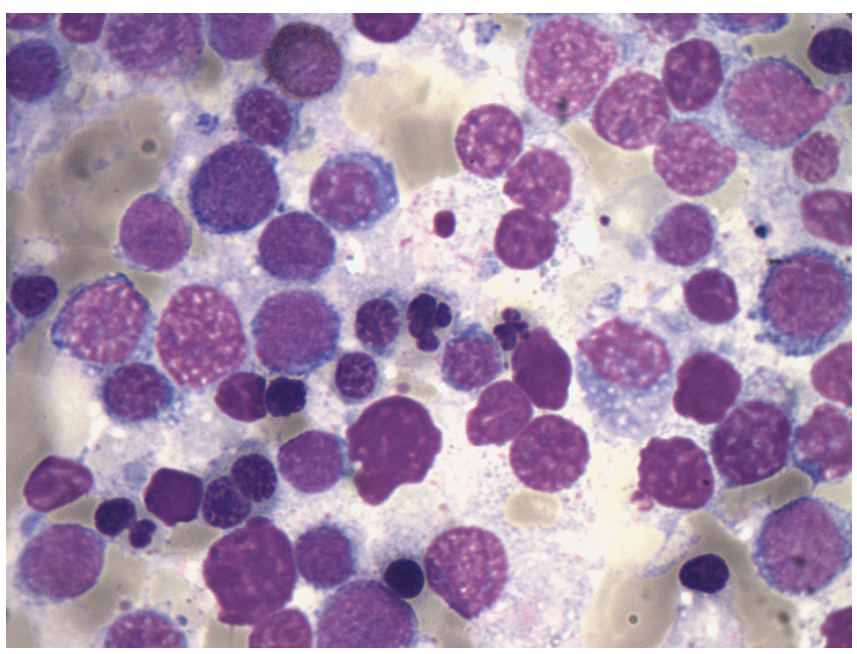

Figure 2: Bone marrow aspirate showing a hypercellular marrow with erythroid hyperplasia and dyserythropoiesis in the form of nuclear budding and nuclear membrane irregularities (Wright Giemsa stain, x100)

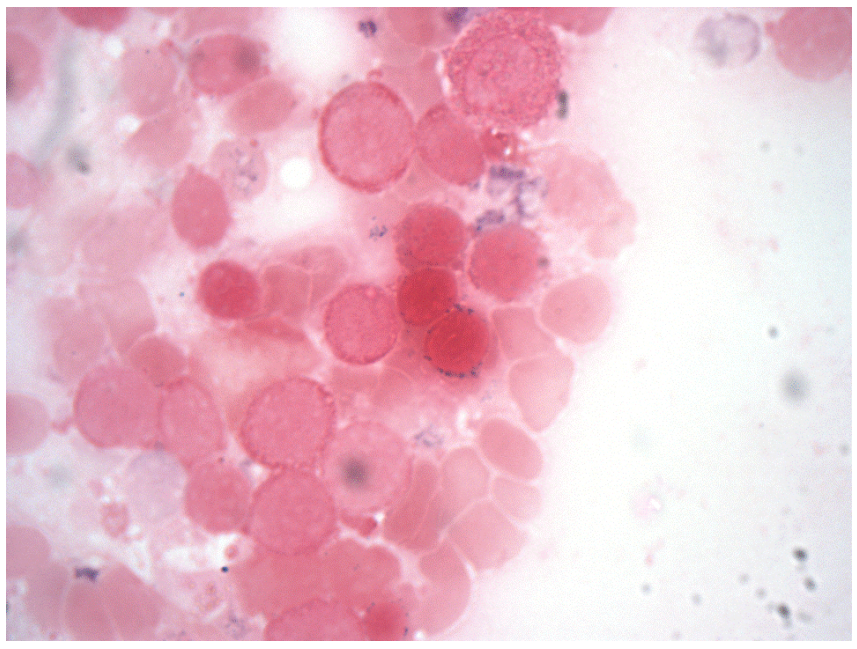

Figure 3: Ringed sideroblasts. (Iron stain, x100) 


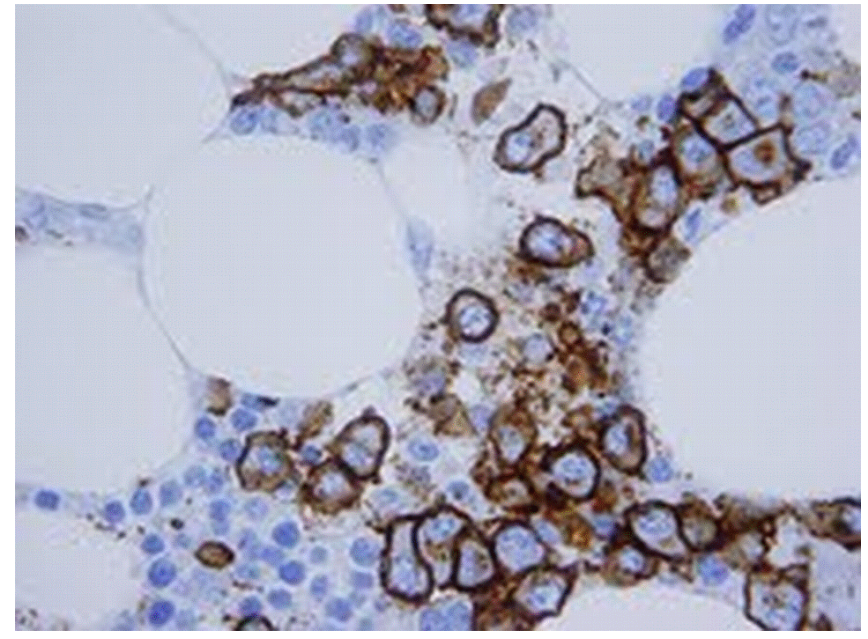

Figure 2: CD138 immunostain highlighting myeloma cells present in clusters in the core biopsy (x40)

showed an elevated IgA ( $777 \mathrm{mg} / \mathrm{dl})$ with a significant increase in kappa free light chains $(246 \mathrm{mg} / \mathrm{dl})$, serum protein electrophoresis results were adversely affected by the elevated bilirubin. Serum immunofixation studies showed a monoclonal IgA kappa. A radiologic full body-bone survey showed no lytic lesions.

The patient was started on treatment for his plasma cell myeloma with bortezomib and dexamethasone. However he experienced a rapid worsening in his liver function as well as progressive anuric renal failure. The patient expired in the hospital three weeks after admission. An autopsy was not performed.

\section{DISCUSSION}

Our patient was diagnosed with simultaneous de novo multiple myeloma, systemic amyloidosis and MDS-RARS. The overall annual incidence of MDS is 3$>20$ per 100,000, with a median age of 70 years and a male predominance [2]. MM is a common hematologic malignancy representing $10-15 \%$ of all hematologic neoplasms. It is also frequently seen in older males (median age 70 years) [3]. Therapy-related MDS following chemotherapy for MM is well recognized and four to ten percent of patients with MM develop MDS as a complication of therapy [1]. However, there have been only rare reports of MM and MDS coexisting as de novo processes. MDS is a clonal stem cell disorder while MM results from a clonal expansion of an immunoglobulin secreting, heavy-chain classswitched, terminally differentiated B cell. The question whether these two distinct hematological diseases are independent processes that coexist by chance or whether one induces the other remains open.

Mufti et. al. [4] proposed that in some patients with MM, unstable haematopoietic clones are present and these clones can develop into myeloid neoplasms leading to simultaneous de novo diseases involving both myeloid and lymphoid lineages. Tsiara et. al. [1] proposed that malignant transformation of a single precursor cell may have the capability to differentiate into both lymphoid and myeloid neoplasms as the possible etiology, a theory that Mufti et al found unlikely. This theory is partly supported by the fact that common cytogenetic abnormalities have been detected in MDS and MM such as deletion of chromosome 13. Copplestone et. al. [5] suggested that either myeloid neoplasm or lymphoid neoplasm could release growth factors that affected the other cell line. This is supported by the findings of Sefer et. al. [6] who demonstrated elevated IL-6 in both diseases. In addition, neoplastic plasma cells are capable of secreting cytokines such as vascular endothelial growth factor (VEGF) that support the self-renewal of cytogenetically abnormal hematopoietic clones in the bone marrow and these clones can eventually progress to MDS [7]. The fact that B-lymphoid and myeloid malignancies co-exist quite frequently suggests a nonfortuitous association.

\section{CONCLUSION}

Concurrent de novo disease processes involving both myeloid and lymphoid cell lines may not be as rare as they seem. Cytopenias in patients with MM should not be attributed to the disease alone but a thorough investigation for an associated concurrent myeloid disorder should be undertaken. Our patient's anemia that required a blood transfusion was probably caused by both marrow infiltration by plasma cells and MDS-RARS. Presence of concurrent MDS would influence the choice of therapy and these patients need to be watched closely for progression of the myeloid disorder. Our patient unfortunately succumbed to multi-organ failure secondary to systemic amyloidosis.

$* * * * * * * * *$

\section{Author Contributions}

Sangeeta Mehendale - Conception and design, Acquisition of data, Analysis and interpretation of data, Drafting the article, Critical revision of the article, Final approval of the version to be published

Pritesh Patel - Conception and design, Acquisition of data, Analysis and interpretation of data, Drafting the article, Critical revision of the article, Final approval of the version to be published

Valerie Lindgren - Conception and design, Acquisition of data, Analysis and interpretation of data, Drafting the article, Critical revision of the article, Final approval of the version to be published

Damiano Rondelli - Conception and design, Acquisition of data, Analysis and interpretation of data, Drafting the article, Critical revision of the article, Final approval of the version to be published 
Sujata Gaitonde - Conception and design, Acquisition of data, Analysis and interpretation of data, Drafting the article, Critical revision of the article, Final approval of the version to be published

\section{Guarantor}

The corresponding author is the guarantor of submission.

\section{Conflict of Interest}

Authors declare no conflict of interest.

\section{Copyright}

(C) Sujata Gaitonde et. al. 2010; This article is distributed under the terms of Creative Commons attribution 3.0 License which permits unrestricted use, distribution and reproduction in any means provided the original authors and original publisher are properly credited. (Please see www.ijcasereportsandimages.com /copyright-policy.php for more information.)

\section{REFERENCES}

1. Tsiara S, Economou G, Panteli A, Isaakidis P, Kapsali E and Bourantas K.L. Coexistence of myelodysplastic syndrome and multiple myeloma. J Exp Clin Cancer Res 1999;18:565-566.

2.

3. Jemal A, Siegel R, Ward E, Murray T, Xu J, Thun MJ. Cancer statistics. CA Cancer J Clin 2007;57:4366.

4. Mufti, GJ, Hamblin TJ, Clein GP, Race C. Coexistent myelodysplasia and plasma cell neoplasia. Br J Haematol 1983;54:91-96.

5. Copplestone JA, Mufti GJ, Hamblin TJ, Oscier DG. Immunologic abnormalities in myelodysplastic syndromes II. Coexistent lymphoid or plasma cell neoplasms: a report of 20 cases unrelated to chemotherapy. Br J Haematol 1986;63:149-159.

6. Sefer D, Marisavljevic D, Bogdanovic A, Andrejevic S, Boskovic D, Suvajdid N, Colovic M. Circulating Interleukin-6 (IL-6) in patients with myelodysplastic syndromes. Leukemia Res 1999;23:78.

7. Bellamy WT, Richter L, Sirjani D, Roxas C, Glinsmann-Gibson B, Frutiger Y, Grogan TM, List AF. Vascular endothelial growth factor is an autocrine promoter of abnormal localized immature myeloid precursors and leukemia progenitor formation in myelodysplastic syndromes. Blood. 2001;97:1427-1434. 